Open Access

\title{
Age dependent normal horizontal VOR gain of head impulse test as measured with video-oculography
}

\author{
Benjamin Mossman ${ }^{1 \dagger}$, Stuart Mossman ${ }^{1 * \dagger}$, Gordon Purdie $^{2}$ and Erich Schneider ${ }^{3}$
}

\begin{abstract}
Background: The head impulse test (HIT) is a recognised clinical sign of the high frequency vestibulo-ocular reflex (VOR), which can be quantified with video-oculography. This measures the VOR gain as the ratio of angular eye velocity to angular head velocity. Although normative data is available for VOR gain with video-oculography, most normal studies in general include small numbers of subjects and do not include analysis of variation of VOR gain with age. The purpose of our study was to establish normative data across 60 control subjects aged 20 to 80 years to represent a population distribution.
\end{abstract}

Methods: Sixty control subjects without any current or previous form of brain disorder or vertigo participated in this study and form the basis for future comparison to patients with vestibular lesions. The relationship between the horizontal vestibulo-ocular reflex (HVOR) velocity gain and age was analysed using a mixed regression model with a random effect for subjects. Differences in testing technique were assessed to ensure reliability in results.

Results: The mean HVOR velocity gain of 60 normal subjects was $0.97(S D=0.09)$ at $80 \mathrm{~ms}$ and $0.94(S D=0.10)$ at $60 \mathrm{~ms}$. The 2 SD lower limit of normal HVOR velocity gain was 0.79 at $80 \mathrm{~ms}$ and 0.75 at $60 \mathrm{~ms}$. No HVOR velocity gain fell below 0.76 and 0.65 at $80 \mathrm{~ms}$ and $60 \mathrm{~ms}$ respectively. The HVOR velocity gain declined by 0.012 and 0.017 per decade as age increased at $80 \mathrm{~ms}$ and $60 \mathrm{~ms}$ respectively. A non-physiologically high horizontal HVOR velocity gain was found to occur in tests where passive HITs were predictable in direction and time and where target distance was below $0.70 \mathrm{~m}$.

Conclusions: Normative data with respect to HVOR velocity gain decreases slightly with age, but with careful attention to methodology the 2 SD lower limit of normal is relatively robust across a wide age range and into the eighth decade, without requirement for adjustment with age.

Keywords: Head impulse test, Horizontal vestibulo-ocular reflex, Semicircular canal, Eye movements

\section{Background}

The horizontal head impulse test (HIT) is a well recognised clinical tool to test the horizontal vestibular ocular reflex (HVOR). The subject maintains fixation on an object straight ahead while sudden head impulses are applied in the horizontal angular plane and eye movements are observed for catch up saccades [1]. If a subject's vestibular ocular reflex (VOR) is normal, the eyes should remain

\footnotetext{
* Correspondence: stuart.mossman@ccdhb.org.nz

${ }^{\dagger}$ Equal contributors

'Department of Neurology, Wellington Hospital, Riddiford Street, Private Bag 7902, Wellington South, Wellington, New Zealand

Full list of author information is available at the end of the article
}

focused on the fixation target during head rotation. However, if there is a significant semicircular canal deficit on the side corresponding to rotation, the ipsilateral VOR response will be inadequate and a significant catch up saccade(s) may be seen.

Video-oculography (VOG) goggles allow quantitative recording of the eye and head movements during the HIT. Covert saccades (occurring during the head movement, usually undetectable clinically) and overt saccades (occurring after the head movement and detectable clinically) are both recorded by the camera. VOG also provides a quantitative measure of the VOR deficit, distinguishing 
abnormal from normal subjects. An advantage of the video HIT (vHIT) over search coils [2] is that it is less invasive, has simple setup and is readily available for clinicians. This relatively new technology uses a high speed compact camera (220 Hz sampling rate) that is attached to lightweight goggles (EyeSeeCam HIT) [3]. The system is used in conjunction with computer software to track pupil movement. Head movement is recorded through a motion sensor attached to the goggles [3-5]. A velocity gain is calculated by dividing instantaneous eye velocity by instantaneous head velocity. The objective was to obtain HVOR velocity gain data to represent a population distribution of normal subjects.

\section{Methods}

The study used quantitative recordings with VOG to measure the HVOR velocity gain during the high frequency horizontal HIT. Sixty three normal subjects were tested (10 per decade, ranging from 20 to 80 years). Exclusion criteria, ascertained at the time of recruitment were no previous form of brain disorder, vertigo, or restricted neck movement.

Before testing, each subject was given verbal and written information regarding the test procedure and rationale. This outlined the risks and exclusion criteria, with signed consent required. As the study established normative data, approval was not required by our Central Regional Ethics Committee.

\section{Experimental procedure}

Before formal testing, we ensured that the subject managed an adequate range of unrestricted, painless angular head rotation. The subject was seated $1.5 \mathrm{~m}$ directly in front of a fixation target at eye level. VOG goggles were fitted tightly to the subject's head to reduce goggle slippage. The camera was focused on the eye while the subject fixated on the target. The subject was instructed to keep his/her eyes open widely so as not to obscure the pupil. If the palpebral fissure remained unduly narrowed, including from ptosis with redundant skin folds or long eye lashes, the eyelids were held open by the rims of the goggles. Even though this procedure can alter the vertical offset of the calibration parameters, it has no effect on the scaling of the calibration. The HVOR velocity gain therefore remains unaffected.

The system was calibrated with the subject altering fixation around five dots, $8.5^{\circ}$ apart, projected onto the wall in front of them. The dots were emitted from a goggle-mounted laser and a diffraction grating [6]. The fixation sequence was arbitrary and the subject was instructed to spend no more than one second fixating on each dot. If errors occurred, the operator could repeat the calibration procedure.

The testing method outlined to the subjects included that they should:
- clench their teeth during the HIT to reduce jaw movement and facilitate a more direct force transfer to the head and reduce movement artefact

- maintain a relaxed neck musculature and not anticipate or aid in head movements

- not move the goggles once calibration was completed

- keep their eyes open wide and minimise blinking to allow the software to keep precise track of pupil movements

- maintain gaze on the fixation target throughout the testing procedure of angular head rotation

Eye and head rotations were measured during the HIT while the examiner manually applied rapid unpredictable (in direction and time) angular head rotations (peak head velocity $150 \%$ to $300 \%$ s) [7]. Instantaneous HVOR velocity gains were calculated by the EyeSeeCam VOG software at $80 \mathrm{~ms}$ and $60 \mathrm{~ms}$ [8]. Head accelerations were manually controlled so that peak head velocity would occur at $80 \pm 15 \mathrm{~ms}$ into each rotation [9]. This was achieved with angular head displacements of small amplitude $\left(6^{\circ}-12^{\circ}\right)$ and rapid rotation. Rotation of the subject's head was performed with the examiner standing behind the seated subject. Six to ten unpredictable head rotations in both directions were performed from a central head position. This sequence was repeated twice to ensure adequate data collection and a check for data consistency, but more frequently if a subject needed additional training or if results were affected by artefact. Head rotations were achieved by the examiner firmly holding the mandible with three fingers clasped below and a thumb and forefinger above the jaw line (Fig. 1). This reduced skin movement and thus goggle slippage, decreasing the amount of artefact. Care was taken to avoid touching the goggles strap during head rotation. A single examiner ${ }^{\mathrm{BM}}$ performed all tests throughout the study.

\section{Data analysis}

Data with undue technical artefact (with blinks and obscured pupils) was discarded. This selection was unbiased and not determined by HVOR velocity gain. The graphed HIT sequences for each subject were assessed and the test with the least artefact was selected for analysis of HVOR velocity gain (Figs. 2 and 3 exemplify reliable and artefactual data). Only one of the HIT sequences was used in order to achieve similar properties to those expected from real-world clinical examinations. An average of 3.2 (range 2-6) HIT sequences for each subject were used to calculate repeatability of the test. This method took a pooled standard deviation for the left and right and used a definition for a repeatability coefficient adopted by the British Standards Institution [10]. 


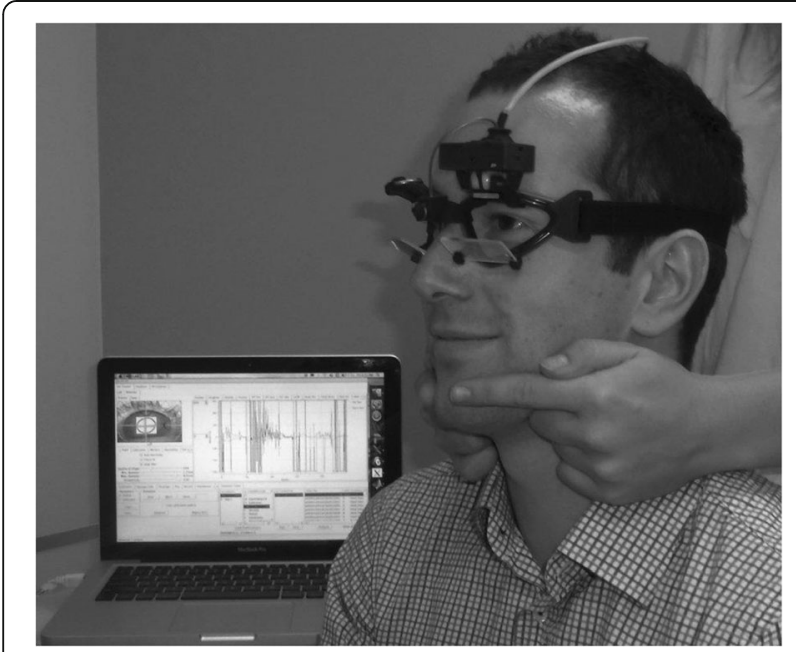

Fig. 1 Method of testing, where the subject's head is firmly held by the mandible, with three fingers clasped below and a thumb and forefinger above the jaw line
HVOR velocity gain at $80 \mathrm{~ms}$ and $60 \mathrm{~ms}$ for both left and right rotations were used to assess the normative range. The $80 \mathrm{~ms}$ time analysis was used in case of artefact occurring in the early stages of head rotation (and attributed to goggle slip) and the $60 \mathrm{~ms}$ time analysis because of the frequent occurrence of covert catch up saccades seen in some patients with vestibular deficits. Two analysis intervals obviate these potential problems and provide an internal check for reliability. Analysis for each head impulse was performed over a $10 \mathrm{~ms}$ window centred at $80 \mathrm{~ms}$ and $60 \mathrm{~ms}$. The median of these values was calculated in order to lessen the weighting from any outliers. Data was manipulated using the EyeSeeCam VOG software backed by Matlab scripts for data analysis. The same scripts were used in another study with search coils [8]. This analysis over a short time window has been recognised as the gold standard [2].

The main purpose of the study was to assess the relationship between HVOR velocity gain and age with analysis using a mixed regression model with a random effect for subjects. Differences between time of HVOR velocity gain analysis $(80 \mathrm{~ms}$ or $60 \mathrm{~ms})$ and sides were compared with analysis of variance with

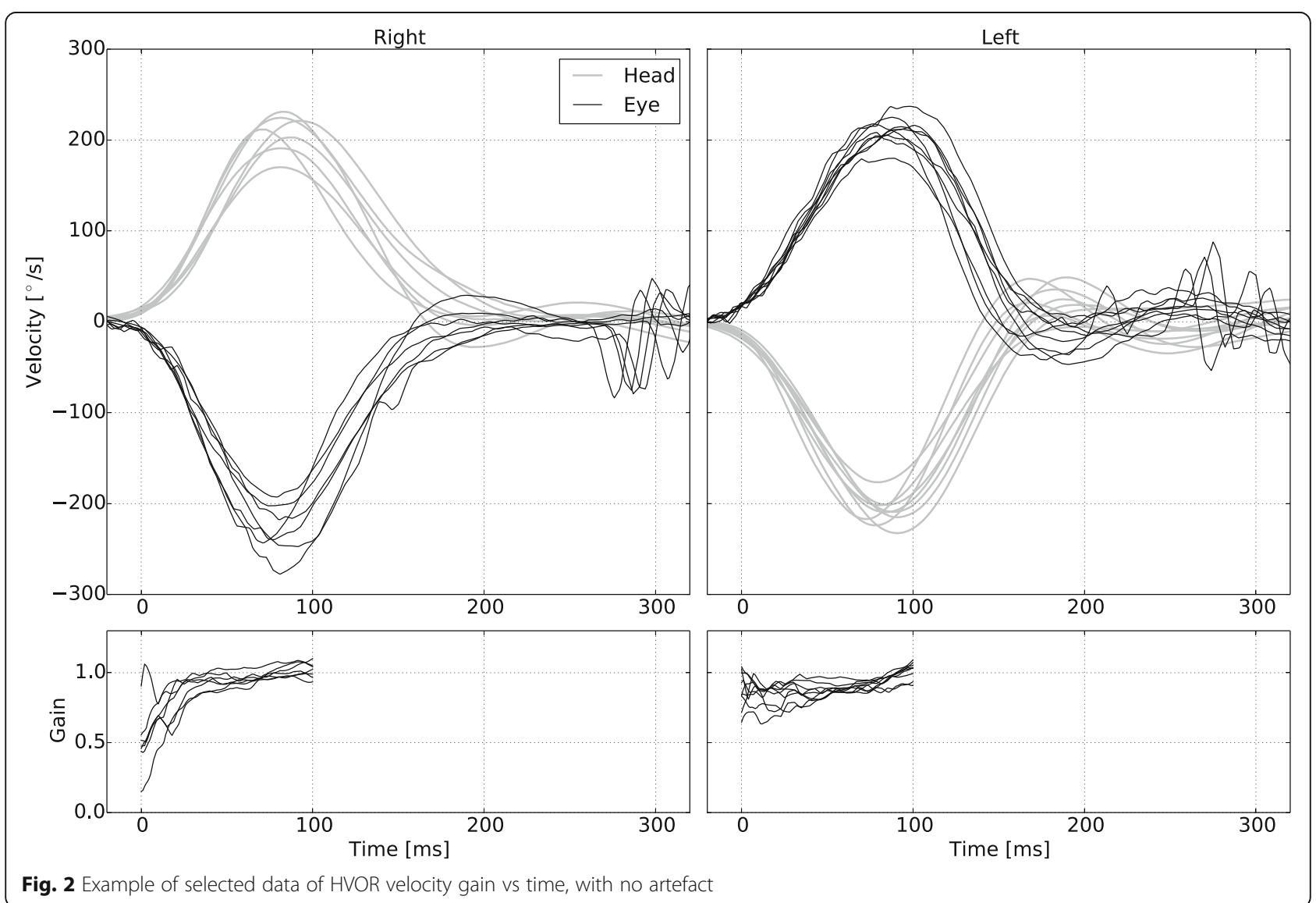



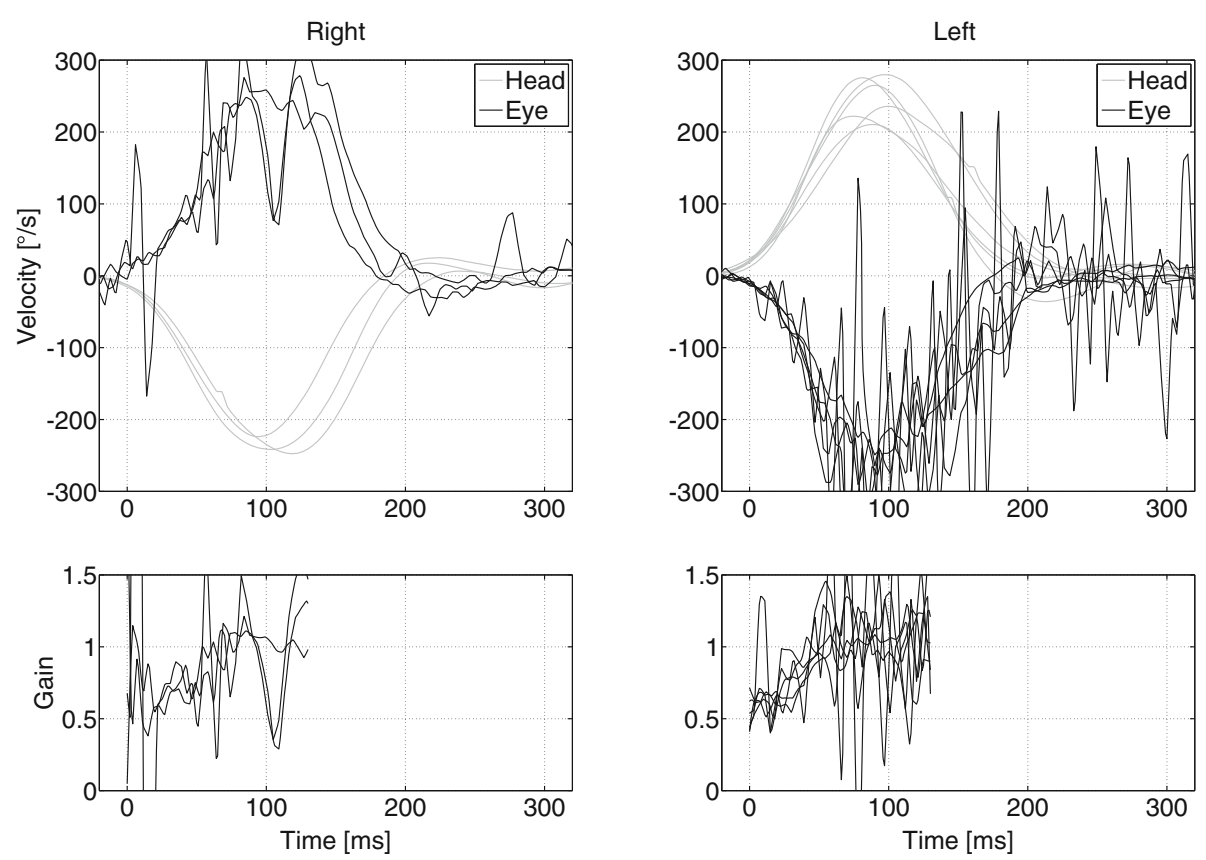

Fig. 3 Example of omitted data of HVOR velocity gain vs time with artefact. In this subject, too few head rotations were plotted, and a narrow palpebral fissure lead to artefact. Nevertheless, the examiner can determine from the observation of the eye and head velocity traces that the vestibular function of this subject is normal. Despite the artefacts, one can observe that peak eye velocity corresponds with peak head velocity

terms for time of velocity gain analysis, side, their interaction and their random terms with subjects.

Additional testing was also performed to assess the effect of fixation distance and predictable versus unpredictable head impulses on data reliability.

Five normal subjects were tested at a series of fixation distances $(0.23,0.40,0.70,1.00,1.30,1.60$ and $1.90 \mathrm{~m})$ to assess the dependence of the HVOR velocity gain on distance. This was analysed using a mixed linear regression with a random term for subject and autoregressive errors with distance.

Eighteen of the normal subjects were tested using both predictable and unpredictable head rotations (in direction and time). Predictable head impulses in direction alternated sequentially between the right and left at a regular time interval. The effect of predictable testing was analysed for variance with terms for side, whether or not the rotation was predictable, a random term for subjects, and interaction terms.

\section{Results}

HVOR velocity gains were obtained for 63 subjects over the six decades from age 20 to 80 . Horizontal head impulses were carried out with peak head velocities ranging from $150 \%$ to $300 \%$ (corresponding to peak head accelerations of $2300 \% / \mathrm{sec}^{2}$ to $5900 \% \mathrm{sec}^{2}$ ). Results in three subjects (5\%) were discarded due to neck stiffness limiting angular head velocity, and artefact attributed to narrowed palpebral fissures (aged $>60$ years). The remaining 60 subjects' left and right HVOR velocity gains were plotted showing a frequency distribution. This included subjects with and without overt saccades as occur in normal subjects clinically. This gave a combined total of 120 HVOR velocity gains at both $80 \mathrm{~ms}$ and $60 \mathrm{~ms}$, as displayed in Fig. $4 \mathrm{a}$ and $\mathrm{b}$.

The distributions of HVOR velocity gain at $80 \mathrm{~ms}$ and $60 \mathrm{~ms}$ were not significantly different from normal distributions (Shapiro-Wilk test, $\mathrm{W}=0.988, p=0.39$ and $\mathrm{W}=0.990, p=0.54$ respectively) around mean values of $0.97(\mathrm{SD}=0.09)$ and $0.94(\mathrm{SD}=0.10), n=120$. The mean (95\% confidence intervals (CI)) HVOR velocity gains to the left and right are both $0.97(0.94-0.99)$ at $80 \mathrm{~ms}$, and $0.94(0.92-0.96)$ to the left and $0.94(0.92-0.97)$ to the right at $60 \mathrm{~ms}$. The lower limit of the normal HVOR velocity gain (2SD below mean) was 0.79 at $80 \mathrm{~ms}$ and 0.75 at $60 \mathrm{~ms}$. The lowest and highest values of the normal HVOR velocity gain were 0.76 and 1.18 at $80 \mathrm{~ms}$ and 0.65 and 1.17 at $60 \mathrm{~ms}$.

The interaction of the time of analysis ( $80 \mathrm{~ms}$ or $60 \mathrm{~ms}$ ) and side was not significant $(p=0.91)$. The HVOR velocity gains were significantly different between $80 \mathrm{~ms}$ and $60 \mathrm{~ms}(0.02 ; 95 \%$ CI $0.04-0.01$; SD $0.05 ; p=0.0004)$. The HVOR velocity gains were not significantly different between the sides $(0.00 ; 95 \%$ CI $-0.02-0.02$; SD $0.08 ; p=$ 0.93). The HVOR velocity gain repeatability coefficients 


\section{a)}

\section{Frequency of normal HVOR velocity gain at $80 \mathrm{~ms}$ in 60 subjects $(n=120)$}

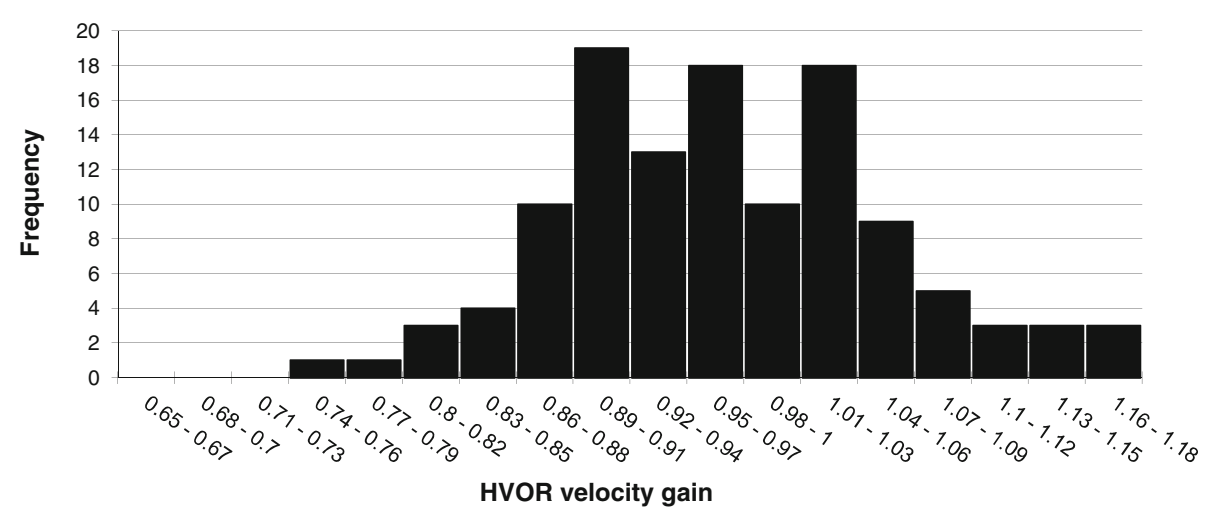

b)

Frequency of normal HVOR velocity gain at $60 \mathrm{~ms}$ in 60 subjects $(n=120)$

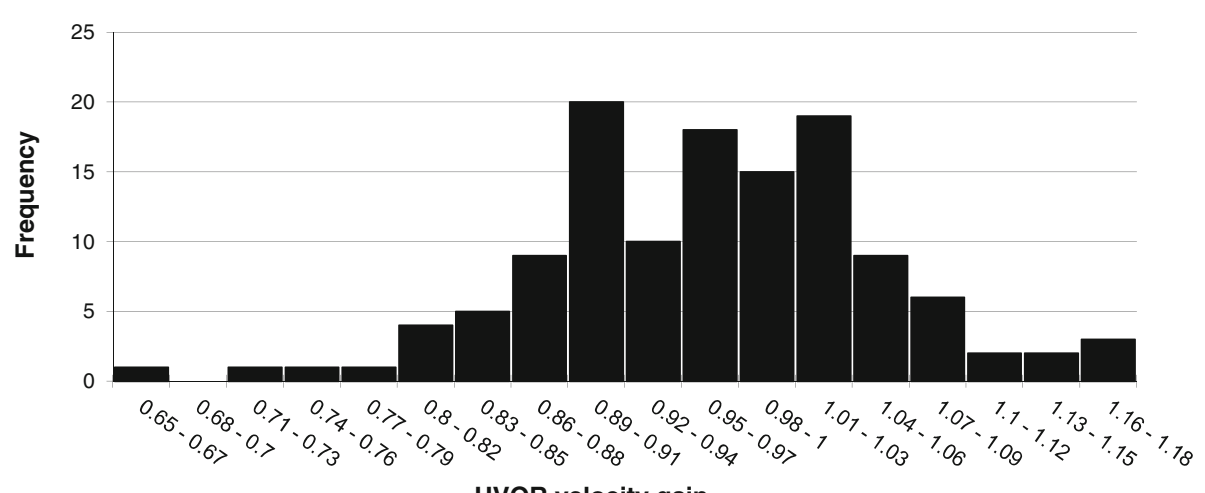

HVOR velocity gain

Fig. 4 a: Normal HVOR velocity gain frequency at $80 \mathrm{~ms}$ across the second to eighth decades during left and right head rotations. Results show a normal distribution (Shapiro - Wilk test for normality, $p=39$ ). HVOR velocity gain is the ratio of angular eye velocity to angular head velocity. $\mathbf{b}$ : Normal HVOR velocity gain frequency at $60 \mathrm{~ms}$ across the second to eighth decades during left and right head rotations. Results show a normal distribution (Shapiro - Wilk test for normality, $p=0.54$ ). HVOR velocity gain is the ratio of angular eye velocity to angular head velocity

were 0.12 at $80 \mathrm{~ms}$ and 0.10 at $60 \mathrm{~ms}$ (95\% of differences are expected to be less than these).

The HVOR velocity gain at $80 \mathrm{~ms}$ declined by 0.012 (95\% CI 0.001 - 0.022) per decade as age increased $(p=0.028)$, and at $60 \mathrm{~ms}$ declined by 0.017 (95\% CI 0.006 - 0.029) per decade as age increased $(p=0.005)$ (Fig. 5a, b). In patients younger than 70 years, the HVOR velocity gain was always above 0.80 at $80 \mathrm{~ms}$ and always above 0.76 at $60 \mathrm{~ms}$.

The normalised HVOR velocity gain asymmetry was the absolute difference between left and right divided by the sum $\times 100$ [11]; the $95^{\text {th }}$ percentile for $80 \mathrm{~ms}$ is 9.2 with a maximum of 12.5 and for $60 \mathrm{~ms}$ is 8.8 with a maximum of 16.7 . There was no significant correlation with age at $80 \mathrm{~ms}$ or $60 \mathrm{~ms}$ with Spearman correlation coefficients of $0.12(p=0.36)$ and $0.11(p=0.42)$ respectively. The un-normalised gain asymmetry, or absolute value of the gain difference between the two sides $[12,13]$, was zero; the $95^{\text {th }}$ percentile for $80 \mathrm{~ms}$ is 0.17 with a maximum 0.26 and for $60 \mathrm{~ms}$ is 0.18 with a maximum 0.26 .

During testing the direction and time of head rotation was unpredictable. In addition, eighteen of the subjects were also tested using a predictable HIT. The mean HVOR velocity gain at $80 \mathrm{~ms}$ was 0.06 (95\% CI 0.01 $0.10 ; p=0.014$ ) higher when testing was carried out in a predictable manner (as high as 1.35). HVOR velocity gain between the left and the right increased similarly with predictable rotations $(p=0.55)$.

Five subjects were also tested for target fixation dependence of the HVOR velocity gain at $80 \mathrm{~ms}$ over increasing distance $(0.23,0.4,0.7,1.0,1.3,1.6$ and $1.9 \mathrm{~m})$. There was an inverse relationship between HVOR velocity gain and distance, with no significant difference between the rate of HVOR velocity gain change for the left and right $(p=0.12)$. With a common slope, the HVOR velocity gain decreased by $13 \%$ (95\% CI $9 \%-17 \%$; $p=0.002$ ) per metre. 


\section{a) Age dependent HVOR velocity gain at $80 \mathrm{~ms}$ in 60 subjects $(n=120)$}

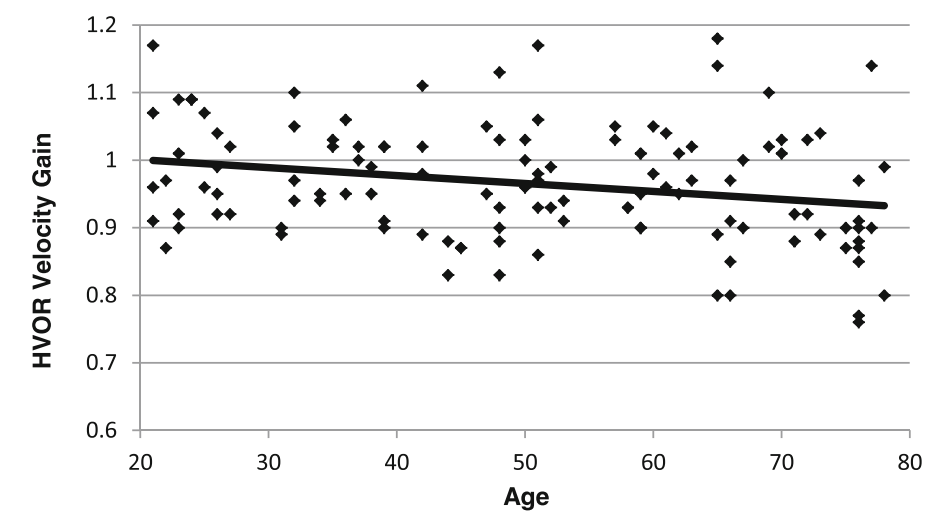

\section{b) Age dependent HVOR velocity gain at $60 \mathrm{~ms}$ in 60 subjects $(n=120)$}



Fig. 5 a: Normal HVOR velocity gain at 80 ms decline with age. HVOR velocity gain was found to decline by 0.012 per decade with increasing age (95\% Cl 0.001 to $0.022 ; p=0.028)$. HVOR velocity gain is the ratio of angular eye velocity to angular head velocity. $\mathbf{b}$ : Normal HVOR velocity gain at $60 \mathrm{~ms}$ decline with age. HVOR velocity gain was found to decline by 0.017 per decade with increasing age $95 \% \mathrm{Cl} 0.006-0.029 ; p=0.005$ ).

HVOR velocity gain is the ratio of angular eye velocity to angular head velocity

\section{Discussion}

In 60 normal subjects aged $20-80$ years, the mean HVOR velocity gain at $80 \mathrm{~ms}$ of 0.97 was little different from that at $60 \mathrm{~ms}$ of 0.94 . The $2 \mathrm{SD}$ lower limit of the HVOR velocity gain at $80 \mathrm{~ms}$ was 0.79 and at $60 \mathrm{~ms}$ was 0.75 . Although statistically significant, the difference in mean HVOR velocity gain, rounded to 0.02 (95\% CI 0.04-0.01) between $80 \mathrm{~ms}$ and $60 \mathrm{~ms}$ is not clinically important. Nevertheless, in patients with an impaired HVOR velocity gain, we think $60 \mathrm{~ms}$ is a more accurate point of measure in the presence of covert saccades than $80 \mathrm{~ms}$. Nonetheless, using both points of measure provides a check on the consistency of results, and the flatness of the gain trajectory in normal subjects confirms the absence of slip (see lower half of Fig 2).

The lowest and highest values of HVOR velocity gain at $80 \mathrm{~ms}$ (0.76 and 1.18 respectively) were very close to those found in another study using a similar
VOG camera system, with eight normal subjects (HVOR velocity gains of 0.75 and 1.2 respectively). Comparable results were found in the same eight subjects using search coils with a lowest and highest HVOR velocity gain of 0.70 and 1.00 [14]. That study analysed HVOR velocity gain over a $40 \mathrm{~ms}$ window centred at peak acceleration, while our analysis is over a $10 \mathrm{~ms}$ window. The mean HVOR velocity gain at $80 \mathrm{~ms}$ of $0.97(\mathrm{SD}=0.09)$ and at $60 \mathrm{~ms}$ of 0.94 $(\mathrm{SD}=0.10)$ for the 60 subjects also sits within a range of HVOR velocity gains observed using the search coil method with comparable accelerations [15].

Earlier studies using vHIT had not referenced the variation of normal HVOR velocity gain with respect to age [14], though recently VOR gain was found to decrease significantly in subjects older than 70 years [16] or 80 years [17]. In our study, HVOR velocity gain at $80 \mathrm{~ms}$ 
and $60 \mathrm{~ms}$ declined by 0.012 and 0.017 respectively per decade as age increased. This decline in HVOR velocity gain with age is consistent with the decline shown in a previous study of the HVOR velocity gain with head impulses using the search coil method [18]. At a practical level, the variation of the HVOR velocity gain with age both with vHIT and search coils was small, justifying the inclusion of our 10 subjects in the eighth decade in normative data, despite a statistically significant decrease in HVOR velocity gain with age. Unlike vestibular evoked myogenic potentials which may be lost with age, our results suggest that the vHIT HVOR velocity gain stands up relatively well with age.

Our highest HVOR velocity gain of 1.18 is not physiological and is likely to relate to goggle slip. This was minimised through firm placement of the examiner's hands, clear from the goggle straps, holding the mandible to reduce slip. However, in the elderly who have looser skin and people with different facial structures or long hair, goggle slippage may still occur. We did not attempt to reduce slippage by using band aids across the nose, or placing dental paste on the nose [19], though these approaches might be considered if an individual subject's slippage affects the interpretation of results.

An adequate head acceleration of $2300 \% \mathrm{sec}^{2}$ to $5900 \% \mathrm{sec}^{2}$ was achieved in each HIT to ensure the detection of a high frequency vestibular deficit [13, 18]. This was monitored in real time by ensuring a peak head velocity of $150 \%$ to $300 \%$ at $80 \mathrm{~ms}$ with low amplitude head movements.

Repeatability at $80 \mathrm{~ms}$ and $60 \mathrm{~ms}$ revealed consistent results across all four tests carried out on each subject. This verifies that in the absence of artefact, only one sequence of $6-10$ sets of rotation in each direction is necessary for clinical interpretation of the HVOR.

It is important to ensure that the method of the HIT is unpredictable in direction and time. Testing in a predictable method confirmed a non-physiological abnormally high HVOR velocity gain. This may result from pre-programming which can augment the VOR and enhance the HVOR velocity gain [20,21].

Analysis was carried out at a fixation target distance of $1.50 \mathrm{~m}$. HVOR velocity gain depends on target distance. As target distance was decreased, HVOR velocity gain increased, consistent with findings during search coil testing $[22,23]$. In our study, the major influence of target distance on HVOR velocity gain appeared to occur at distances of less than $0.70 \mathrm{~m}$. Comparable HVOR velocity gains were found between our study (testing at a $1.50 \mathrm{~m}$ fixation target distance) and another study (testing at a $0.91 \mathrm{~m}$ fixation target distance) [14]. The dependency of the HVOR velocity gain on target distance is due to the different topography of the axes of rotation of the eyes and head [23].

VOG overcomes the clinical problem of a false negative HIT due to covert saccades and allows discrimination between physiological and pathological overt saccade(s). A clinical application is that in acute vertigo with clear impairment of HVOR velocity gain and the absence of CNS symptoms or signs, neuroimaging is not required; while the acutely vertiginous subject with a normal HVOR velocity gain needs consideration of a CNS cause or inferior vestibular neuritis [24]. The results of this study allow a comparison to be drawn between normal subjects and patients with vestibular lesions, across adult age groups to 80 years.

\section{Conclusions}

The mean HVOR velocity gain of 60 normal subjects was $0.97(\mathrm{SD}=0.09)$ at $80 \mathrm{~ms}$ and $0.94(\mathrm{SD}=0.10)$ at $60 \mathrm{~ms}$. Despite a significant variation in the HVOR velocity gain with age, these changes are minor, declining by 0.012 and 0.017 per decade as age increased at $80 \mathrm{~ms}$ and $60 \mathrm{~ms}$ respectively, justifying the same normative data for the second to the eighth decades. Normative data at $60 \mathrm{~ms}$ provides an opportunity for assessment when the $80 \mathrm{~ms}$ time interval result may be affected by the appearance of catch-up covert saccades.

\section{Abbreviations \\ HIT: Horizontal head impulse test; HVOR: Horizontal vestibulo-ocular reflex; VHIT: video HIT; VOG: Video-oculography; VOR: Vestibulo-ocular reflex.}

\section{Competing interest}

The development of EyeSeeCam vHIT was supported by funds from the German Federal Ministry of Education and Research under the Grant code 01 EO 0901. Erich Schneider is a salaried member of the Brandenburg University of Technology Cottbus - Senftenberg. He also acts as an unpaid consultant and has received funding for travel from Interacoustics, distributor of EyeSeeCam. He is the general manager and a shareholder of EyeSeeTec $\mathrm{GmbH}$, manufacturer of EyeSeeCam.

Dr Stuart Mossman's and Benjamin Mossman's research project salaries were paid through research funds from Wellington Hospital.

Gordon Purdie is a salaried member of Dean's Department, University of Otago, Wellington.

\section{Authors' contributions}

Study design, analysis, interpretation, drafting or revising of the paper involved all authors. Data was collected by BM alone. Statistical advice and analysis was performed by GP. All authors have given final approval of the version to be published and agree to be accountable for all aspects of the work in relation to accuracy or integrity of the paper.

\section{Acknowledgements}

Thank you to all the Wellington Hospital staff and members of the public who participated as normal subjects in the study.

\section{Author details}

${ }^{1}$ Department of Neurology, Wellington Hospital, Riddiford Street, Private Bag 7902, Wellington South, Wellington, New Zealand. 'Dean's Department, University of Otago, Wellington, New Zealand. ${ }^{3}$ Institute of Medical Technology, Brandenburg University of Technology Cottbus, Senftenberg, Germany.

Received: 13 February 2015 Accepted: 18 June 2015

Published online: 04 July 2015

\section{References}

1. Halmagyi GM, Cuthroy IS. A clinical sign of canal paresis. Arch Neurol. 1988;45:737-9.

2. Aw S, Haslwanter T, Halmagyi G, Curthoys I, Yavor R, Todd MM. Three-dimensional vector analysis of the human vestibuloocular reflex in 
response to high-acceleration head rotations. I. Responses in normal subjects. J Neurophysiol. 1996;76(6):4009-20.

3. Bartl K, Lehnen N, Kohlbecher S, Schneider E. Head impulse testing using video-oculography. Ann NY Acad Sci. 2009;1164:331-3.

4. Hofmann P, Kellig A, Hoffmann H-U, Dara RG. Vestibular equipment onboard MIR. Acta Astronaut. 1998;43(3-6):313-9.

5. Strupp M, Glasauer S, Jahn K, Schneider E, Krafczyk S, Brandt T. Eye movements and balance. Ann NY Acad Sci. 2003;1004:352-8.

6. Pelz JB, Canosa R. Oculomotor behavior and perceptual strategies in complex tasks. Vision Res. 2001;41:3587-96.

7. Halmagyi GM, Weber KP, Aw ST, Todd MJ, Curthoys IS. Impulsive testing of semicircular canal function. Prog Brain Res. 2008;171:187-94.

8. Glasauer S, Lindeiner H, Siebold C, Buttner U. Vertical vestibular responses to head impulses are symmetric in downbeat nystagmus. Neurology. 2004;63:621-5

9. Weber KP, MacDougall HG, Halmagyi GM, Curthoys IS. Impulsive testing of semicircular-canal function using video-oculography. Ann NY Acad Sci. 2009;1164:486-91.

10. Bland JM, Altman DG. Statistical methods for assessing agreement between two methods of clinical measurement. Lancet. 1986;327:307-10.

11. Schmid-Priscoveanu A, Boehmer A, Obzina H, Straumann D. Caloric and search-coil head impulse testing in patients after vestibular neuritis. J Assoc Res Otolaryngol. 2001;2:72-8.

12. Heuberger M, Saglam M, Todd N,Klaus Jahn K, Schneider E, Lehnen N. Covert Anti-Compensatory Quick Eye Movements during Head Impulses. PLoS ONE. 9(4): e93086. doi:10.1371/journal.pone.0093086

13. Weber KP, Aw ST, Todd MJ, McGarvie LA, Curthoys IS, Halmagyi GM. Horizontal head impulse test detects gentamicin vestibulotoxicity. Neurology. 2009;72:1417-24.

14. MacDougall HG, Weber KP, McGarvie LA, Halmagyi GM, Curthoys IS. The video head impulse test, diagnostic accuracy in peripheral vestibulopathy. Neurology. 2009;73:1134-41.

15. Weber KP, Aw ST, Todd MJ, McFarvie LA, Curthoys IS, Halmagyi GM. Head impulse test in unilateral vestibular loss, vestibulo-ocular reflex and catch up saccades. Neurology. 2008;70:454-63.

16. Matiño-Soler E, Esteller-More E, Martin-Sanchez JC, Martinez-Sanchez JM, Perez-Fernandez N. Normative data on angular vestibulo-ocular responses in the yaw axis measured using the video head impulse test. Otol Neurotol. 2015;36(3):466-71.

17. Li C, Layman AJ, Geary R, Anson E, Carey JP, Ferrucci L, et al. Epidemiology of Vestibulo-Ocular Reflex Function: Data from the Baltimore Longitudinal Study of Aging. Otol Neurotol. 2015;36(2):267-72.

18. Brzenzy R, Glasauer S, Bayer O, Siebold C, Buttner U. Head impulses in three orthogonal planes, influence of age. Ann NY Acad Sci. 2003;1004:473-7.

19. Versino $\mathrm{M}$ et al. Artifact avoidance for head impulse testing. Clin Neurophysiol. 2014;125:1071-3.

20. Della Santina CC, Cremer PD, Carey JP, Minor LB. Comparison of head thrust test with head autorotation test reveals that the vestibulo-ocular reflex is enhanced during voluntary head movements. Arch Otolaryngol Head Neck Surg. 2002;128:1044-54.

21. Black RA, Halmagyi GM, Thurtell MJ, Todd MJ, Cuthroys IS. The active head-impulse test in unilateral peripheral vestibulopathy. Arch Neurol. 2005;62(2):290-3.

22. Viirre $E$, Tweed $D$, Milner $K$, Vilis T. A reexamination of the gain of the vestibuloocular reflex. J Neurophysiol. 1986;56(2):439-50.

23. Collewijn H, Smeets JBJ. Early components of the human vestibulo-ocular response to head rotation: latency and gain. J Neurophysiol. 2000;84:376-89.

24. Halmagyi GM, Aw ST, Karlberg M, Curthoys IS, Todd MJ. Inferior vestibular neuritis. Ann NY Acad Sci. 2002;956:306-13.

\section{Submit your next manuscript to BioMed Central and take full advantage of:}

- Convenient online submission

- Thorough peer review

- No space constraints or color figure charges

- Immediate publication on acceptance

- Inclusion in PubMed, CAS, Scopus and Google Scholar

- Research which is freely available for redistribution

Submit your manuscript at www.biomedcentral.com/submit 